Military Technical College Kobry El-Kobbah, Cairo, Egypt

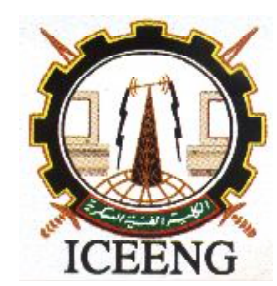

\section{$10^{\text {th }}$ International Conference on Electrical Engineering ICEENG 2016}

\title{
Radiating/One Way Non-Radiating Currents
}

$$
\text { By }
$$

E. A. M. El Fayome *

A. H. Kamel ${ }^{\ddagger}$

H. S. ElHennawy \#

\section{Abstract:}

A new formulation for solving Maxwell's equations is presented. It is based on combining radiating/one way non-radiating currents with volume integral equations. The results of the proposed scheme are compared with those for a number of cases with known analytical solutions. The accuracy and convergence properties of the proposed scheme are reported and the advantages and limitations are detailed.

\section{Keywords:}

Radiation, scattering and radiating/non-radiating current

* Ministry of Interior

\$ Advanced Industrial, Technical and Engineering Center

\# Electronics and Communications Department, Faculty of Engineering, Ain Shams University 


\section{Introduction:}

A new semi-analytical formulation for the computation of the scattered electromagnetic fields from vertically stratified lossy perfect dielectric media is presented. We developed this approach to overcome some of the limitations and difficulties of the existing approaches (Finite-Difference Time-Domain (FDTD), Finite Element Method (FEM), Boundary Element Method (BEM), ... [1-3]). Those limitations include numerical dispersion and numerical polarization, the need to truncate the computational domain, hence, the introduction of unphysical nonreflecting boundaries, the difficulty of representing highly localized sources, the bad accuracy near points where the field is singular, difficulty in dealing with thin layers and other domain parts with fast varying medium properties, inaccuracy of representing evanescent fields and singular and hypersingular integrals (in the BEM).

\section{Formulation:}

The derivation of the scattered electromagnetic field proceeds through three steps. In the first step, the Maxwell's equations are recast in the form of a volume integral equation on the induced electric polarization current inside the scattering lossy perfect dielectric medium. The volume integral equation method has established itself as a reliable formulation approach for scientific problems and engineering applications related to wave phenomena [4].

In the second step, the polarization current is decomposed into radiating and one way (the back scattering direction) non-radiating components that are shown to form a complete orthonormal set of eigen functions. The polarization current inside the scatterer is then expanded in terms of the deduced complete set; the orthogonality of the complete set is used to recast the volume integral equation into a linear system; the expansion coefficients are found by solving the linear system.

In the third step, the back scattered electromagnetic field is computed by applying Green's function [5] to the found expansion coefficient of the radiating part of the polarization current.

\section{A) Volume Integral Equation}

Consider an infinite unphased electric line source oriented along the $z$-direction in free space (or a background medium) with a 2-D inhomogeneity of finite support located in the background medium. The geometry as well as the physical parameters of the inhomogeneity are $z$-independent too. We want to compute the field scattered from the 
inhomogeneity back to the background medium. For a harmonic time dependence of the form $\exp (-i \omega t)$, Maxwell's equations inside the scatterer read

$\nabla \times H(x, y)=-i \omega \dot{o}_{0} \grave{o}_{r}(x, y) E(x, y)+\sigma(x, y) E(x, y)$,

$\nabla \times E(x, y)=i \omega \mu_{0} \mu_{r}(x, y) H(x, y)$,

where $E(x, y)$ is the electric field, $H(x, y)$ is the magnetic field, $\grave{\mathrm{o}}_{r}(x, y)$ is the relative permittivity, $\sigma(x, y)$ is the conductivity, $\mu_{r}(x, y)$ is the relative permeability, of the scatterer with $\grave{o}_{0}$ is the permittivity of free space, $\mu_{0}$ is the permeability of free space (taken as the background medium without loss of generality). The operating frequency is given by $\omega /(2 \pi)$.

Meanwhile, in free space, the Maxwell's equations are given by

$\nabla \times H(x, y)=-i \omega \grave{\mathrm{o}}_{0} E(x, y)+J(x, y)$,

$\nabla \times E(x, y)=i \omega \mu_{0} H(x, y)-M(x, y)$,

where $J(x, y)$ is an electric current source and $M(x, y)$ is a magnetic current source, hence, the scatterer presence in free space can be thought of as an electric polarization, $J(x, y)$, and a magnetic polarization, $M(x, y)$, currents given by

$J(x, y)=\left(\sigma(x, y)-i \omega \grave{o ̀ j}_{r}((x, y)-1)\right) E(x, y)$,

$M(x, y)=-i \omega \mu_{0}\left(\mu_{r}(x, y)-1\right) H(x, y)$.

In the perfect dielectric case, $\mu_{r}(x, y)=1$, hence, $M(x, y)=0$.

Therefore, for such a $z$-independent problem and no magnetic currents, the nonvanishing field components are the electric field in the $z$-direction and the magnetic fields in the $x$ and $y$ directions. The electric field inside the scatterer is given by [4-5]

$$
\begin{aligned}
& E_{z s}(x, y)=E_{z}^{0}(x, y)-\frac{\omega \mu_{0}}{4} \int_{0}^{L} d y^{\prime} \int_{-\infty}^{\infty} d x^{\prime} J_{z}\left(x^{\prime}, y^{\prime}\right) H_{0}^{(1)}\left(x, y ; x^{\prime}, y^{\prime}\right), \\
& E_{z}^{0}(x, y)=-\frac{\omega \mu_{0}}{4} H_{0}^{(1)}\left(k_{0}\left|\rho-\rho_{s}\right|\right), \\
& H_{0}^{(1)}\left(k_{0}\left|\rho-\rho^{\prime}\right|\right)=\frac{1}{\pi} \int_{-\infty}^{\infty} d k_{x} \frac{\exp \left(i k_{x}\left(x-x^{\prime}\right)+i k_{y}\left|y-y^{\prime}\right|\right)}{k_{y}}, \\
& k_{y}=\sqrt{k_{0}^{2}-k_{x}^{2}} \text { with } \operatorname{Im} k_{y}>0,
\end{aligned}
$$

where $E_{z s}(x, y)$ is the total field inside the slab, $E_{z}^{0}(x, y)$ is the incident electric field, $H_{0}^{(1)}\left(\right.$.) is the Hankel function of zero ${ }^{\text {th }}$ order and first kind, $k_{0}=\omega \sqrt{\grave{\mathrm{o}}_{0} \mu_{0}}$ is the free 
space wave number, $\rho$ and $\rho_{s}$ are the location of an observation point and source point respectively. The double integral is on the geometry of the scatterer with $L$ standing for the thickness of the vertically stratified scatterer along the $y$-direction with infinite extend in the $x$ and $z$ directions. Hence,

$J_{z}(x, y)=\left[\sigma(x, y)-i \omega \grave{o}_{0}\left(\grave{o}_{r}(x, y)-1\right)\right] E_{z s}(x, y)$.

With no lateral variations $\left(\grave{\mathrm{o}}_{r}(x, y)=\grave{\mathrm{o}}_{r}(y)\right.$ and $\left.\sigma(x, y)=\sigma(y)\right)$ and the line source located, without loss of generality, at $x_{s}=y_{s}=0$; taking the Fourier transform on $x$, we obtain for $L \geq y \geq 0$

$J_{z}\left(k_{x}, y\right)=Q(y)\left[\frac{i \exp \left(i k_{y} y\right)}{2 k_{y}}+\int_{0}^{L} d y^{\prime} J_{z}\left(k_{x}, y^{\prime}\right) \frac{i \exp \left(i k_{y}\left|y-y^{\prime}\right|\right)}{2 k_{y}}\right]$,

and

$J_{z}\left(k_{x}, y\right)=0, L<y<0$,

which defines an integral equation on $J_{z}\left(k_{x}, y\right)$ in the $k_{x}-y$ domain, with

$$
\begin{aligned}
& Q(y)=\left\{\sigma(y)-i \omega \grave{o}_{0}\left(\grave{\mathrm{o}}_{r}(y)-1\right)\right\} i \omega \mu_{0} \\
& =k_{2}^{2}(y)-k_{0}^{2}, \\
& k_{2}^{2}(y)=\omega^{2} \mu_{0} \grave{o}_{0} \grave{\mathrm{o}}_{r}(y)+i \omega \mu_{0} \sigma(y) .
\end{aligned}
$$

\section{B) Radiating/Non-radiating Currents}

The concept of decomposing the electric polarization current into a radiating part plus a non-radiating part was introduced in the literature many years ago [6]. A radiating current generates fields inside and outside its domain of existence. A non-radiating current produces fields inside its domain of existence only; the field outside its domain of existence is identically zero. Several authors made use of the concept to tackle the inverse scattering problem; see [7] and references therein.

In the work presented here, we make use of the radiating/non-radiating currents decomposition to solve the Maxwell's equations. For such a solution to be accurate and efficient, one is required to find a function representation for the currents in which they form a complete orthogonal set, hence, represent an arbitrary current.

However, except for simple domains such as the sphere [8] and the cylinder [9], such complete sets have not been constructed. For instance, for the simpler domain of a dielectric slab, under consideration in the work presented here, such a complete 
orthogonal set has not been reported and seems to be very difficult to construct; if it exists at all.

\section{C) Radiating/ One Way Non-radiating Currents}

In order to tackle vertically stratified slabs, we introduce the concept of one way nonradiating currents. That is, for back scattered fields in the domain $y \leq 0$, we aim at constructing a complete orthogonal set of radiating/ back scattering non-radiating currents such that the back scattering non-radiating currents generate fields inside their domain of existence and in the forward scattering domain, $y>L$ while the field in the back scattering domain, $y \leq 0$, is identically zero. Hence [5],

$$
\begin{aligned}
& u_{n}^{-}\left(k_{x}, y\right)=\int_{0}^{L} d y^{\prime} \frac{i \exp \left(i k_{y}\left|y-y^{\prime}\right|\right)}{2 k_{y}} \psi_{n}^{-}\left(k_{x}, y^{\prime}\right) \neq 0, \quad \mathrm{~L} \geq \mathrm{y}>0 \\
& u_{n}^{-}\left(k_{x}, y\right)=\int_{0}^{L} d y^{\prime} \frac{i \exp \left(i k_{y}\left(y-y^{\prime}\right)\right)}{2 k_{y}} \psi_{n}^{-}\left(k_{x}, y^{\prime}\right) \neq 0, \quad y>L, \\
& u_{n}^{-}\left(k_{x}, y\right)=\int_{0}^{L} d y^{\prime} \frac{i \exp \left(i k_{y}\left(y^{\prime}-y\right)\right)}{2 k_{y}} \psi_{n}^{-}\left(k_{x}, y^{\prime}\right)=0, \quad y \leq 0,
\end{aligned}
$$

where $\left\{\psi_{n}^{-}\left(k_{x}, y\right), \quad n= \pm 1, \pm 2, \ldots\right\}$ stands for the set of back scattered non-radiating currents, with $\psi_{0}^{-}\left(k_{x}, y\right)$ standing for the radiating part of the current. In addition, $\left\{u_{0}^{-}\left(k_{x}, y\right), u_{n}^{-}\left(k_{x}, y\right), n= \pm 1, \pm 2, \ldots\right\}$ stands for the fields generated by those currents.

It is easy to see, by direct substitution, that

$\psi_{n}^{-}\left(k_{x}, y\right)=\exp (i 2 \pi n y / L) \exp \left(-i k_{y} y\right)$,

$\psi_{0}^{-}\left(k_{x}, y\right)=\exp \left(-i k_{y} y\right)$,

and the set $\left\{\psi_{0}^{-}\left(k_{x}, y\right), \psi_{n}^{-}\left(k_{x}, y\right), n= \pm 1, \pm 2, \ldots\right\}$ is complete and orthogonal on the domain $L \geq y \geq 0$.

For forward scattered fields in the domain $y \geq L$, we aim at constructing a complete orthogonal set of radiating/ forward scattering non-radiating currents such that the forward scattering non-radiating currents generate fields inside their domain of existence and in the back scattering domain, $y<0$ while the field in the forward scattering domain, $y \geq L$, is identically zero. Hence [5], 


$$
\begin{aligned}
& u_{n}^{+}\left(k_{x}, y\right)=\int_{0}^{L} d y^{\prime} \frac{i \exp \left(i k_{y}\left|y-y^{\prime}\right|\right)}{2 k_{y}} \psi_{n}^{+}\left(k_{x}, y^{\prime}\right) \neq 0, \quad \mathrm{~L}>\mathrm{y} \geq 0 \\
& u_{n}^{+}\left(k_{x}, y\right)=\int_{0}^{L} d y^{\prime} \frac{i \exp \left(i k_{y}\left(y^{\prime}-y\right)\right)}{2 k_{y}} \psi_{n}^{+}\left(k_{x}, y^{\prime}\right) \neq 0, \quad y<0, \\
& u_{n}^{+}\left(k_{x}, y\right)=\int_{0}^{L} d y^{\prime} \frac{i \exp \left(i k_{y}\left(y-y^{\prime}\right)\right)}{2 k_{y}} \psi_{n}^{+}\left(k_{x}, y^{\prime}\right)=0, \quad y \geq L,
\end{aligned}
$$

where $\left\{\psi_{n}^{+}\left(k_{x}, y\right), \quad n= \pm 1, \pm 2, \ldots\right\}$ stands for the set of back scattered non-radiating currents, with $\psi_{0}^{+}\left(k_{x}, y\right)$ standing for the radiating part of the current. In addition, $\left\{u_{0}^{+}\left(k_{x}, y\right), u_{n}^{+}\left(k_{x}, y\right), n= \pm 1, \pm 2, \ldots\right\}$ stands for the fields generated by those currents.

It is easy to see, by direct substitution, that

$\psi_{n}^{+}\left(k_{x}, y\right)=\exp (i 2 \pi n y / L) \exp \left(i k_{y} y\right)$

$\psi_{0}^{+}\left(k_{x}, y\right)=\exp \left(i k_{y} y\right)$,

and the set $\left\{\psi_{0}^{+}\left(k_{x}, y\right), \psi_{n}^{+}\left(k_{x}, y\right), \quad n= \pm 1, \pm 2, \ldots\right\}$ is complete and orthogonal on the domain $L \geq y \geq 0$.

We will concentrate on the back scattering case; the forward scattering case is easily found by analogy.

Now, we make use of the complete orthogonal set into the integral equation on $J_{z}\left(k_{x}, y\right)$ $J_{z}\left(k_{x}, y\right)=J_{0}\left(k_{x}\right) \psi_{0}^{-}\left(k_{x}, y\right)+\sum_{n \neq 0} J_{n}\left(k_{x}\right) \psi_{n}^{-}\left(k_{x}, y\right)$,

with $\left\{J_{0}\left(k_{x}\right), J_{n}\left(k_{x}\right)\right\}$ the expansion coefficients of $J_{z}\left(k_{x}, y\right)$ in terms of the complete orthonormal set. Hence,

$$
\begin{aligned}
& u_{0}^{-}\left(k_{x}, y\right)=\frac{i L}{2 k_{y}}\left(\frac{\sin \left(k_{y} y\right)}{k_{y} L}+\left(1-\frac{y}{L}\right) \exp \left(-i k_{y} y\right)\right), \quad L \geq y>0, \\
& u_{0}^{-}\left(k_{x}, y\right)=\frac{i L}{2 k_{y}} \exp \left(-i k_{y} y\right), \quad y \leq 0,
\end{aligned}
$$




$$
\begin{aligned}
u_{n}^{-}\left(k_{x}, y\right)= & \frac{i}{2 k_{y}}\left[\frac{\exp \left(i\left(\alpha_{n}-k_{y}\right) y\right)-\exp \left(i k_{y} y\right)}{i\left(\alpha_{n}-2 k_{y}\right)}\right] \quad L \geq y>0, n \neq 0, \alpha_{n}=\frac{2 n \pi}{L}, \\
& \left.+\frac{\exp \left(-i k_{y} y\right)\left(\exp \left(i \alpha_{n} L\right)-\exp \left(i \alpha_{n} y\right)\right)}{i \alpha_{n}}\right],
\end{aligned}
$$

leading to expressing the integral equation, for $L \geq y \geq 0$, in the form

$$
\sum_{-N}^{N} J_{n}\left(k_{x}\right) \psi_{n}^{-}\left(k_{x}, y\right)=Q(y)\left[\frac{i \exp \left(i k_{y} y\right)}{2 k_{y}}+\sum_{-N}^{N} J_{n}\left(k_{x}\right) u_{n}^{-}\left(k_{x}, y\right)\right] \text {, }
$$

where $2 N+1$ is the number of the complete set functions retained in the expansion.

Using the point-by-point matching method, we generate a linear system, on $n=0, \pm 1, \pm 2, . ., \pm N\}$, of the form

$$
\begin{aligned}
\sum_{-N}^{N} J_{n}\left(k_{x}\right) \psi_{n}^{-}\left(k_{x}, y_{p}\right)= & Q\left(y_{p}\right)\left[\frac{i \exp \left(i k_{y} y_{p}\right)}{2 k_{y}}\right. \\
& \left.+\sum_{-N}^{N} J_{n}\left(k_{x}\right) u_{n}^{-}\left(k_{x}, y_{p}\right)\right], \quad L>y_{p} \geq 0, p=1,2, \ldots, 2 N+1 .
\end{aligned}
$$

In matrix-vector format, the linear system reads

$M J=c$,

$M_{p n}\left(k_{x}\right)=\psi_{n}^{-}\left(k_{x}, y_{p}\right)-Q\left(y_{p}\right) u_{n}^{-}\left(k_{x}, y_{p}\right)$,

$c_{p}\left(k_{x}\right)=Q\left(y_{p}\right) \frac{i \exp \left(i k_{y} y_{p}\right)}{2 k_{y}}$,

leading to

$$
J\left(k_{x}\right)=M^{-1}\left(k_{x}\right) c\left(k_{x}\right) .
$$

\section{D) Scattered Field Computation}

Once the coefficients $\left\{J_{n}\left(k_{x}\right)\right\}$ are computed, we obtain the spectral distribution of the reflected electric field at the base of the slab, $y=0$, namely,

$E_{z r}\left(k_{x}, 0\right)=i \omega \mu_{0} J_{0}\left(k_{x}\right) u_{0}^{-}\left(k_{x}, 0\right)$. 


\section{Results:}

The results of the proposed scheme are compared with those for a number of cases with known analytical solutions. The comparison is done for a wide range of stratified scatterer sizes, permittivities and conductivities as well as source operating frequencies. We present here the results of comparing with a perfect dielectric homogeneous slab of properties $\grave{o}_{r}$ and $\sigma$, for which case, the spectral back scattered field at the base of the slab, $y=0$, is given by

$$
\begin{aligned}
& E_{z r}\left(k_{x}, 0\right)=\frac{-\omega \mu_{0}}{2 k_{y}} \frac{k_{y}-k_{y s}}{k_{y}+k_{y s}} \frac{1-\exp \left(2 i k_{y s} L\right)}{1-\left\{\frac{k_{y}-k_{y s}}{k_{y}+k_{y s}}\right\}^{2} \exp \left(2 i k_{y s} L\right)}, \\
& k_{y s}=\sqrt{\omega^{2} \mu_{0} \grave{\mathrm{o}}_{\mathrm{o}}+i \omega \mu_{0} \sigma-k_{x}^{2}} .(38)
\end{aligned}
$$

The Figures show the real as well as the imaginary spectral electric field values as represented by (37) compared with the representation in (36) resulting from a linear system of size $2 N+1$. The comparison is made for $\grave{\mathrm{o}}_{r}=2$ and $\sigma=0$, with values of $k_{x} / k_{0}$ ranging from 0:1.1. The solid curves are computed from (36) and the squares are computed from (37). Many more cases can be found in [10]. It can be seen in the figures that for a very small range around $k_{x}=k_{0}$ (the grazing incidence angle situation) the field values are very high, reflecting the presence of $1 / k_{y}$ in (37), which tends to infinity at $k_{x} / k_{0}=1$. Nevertheless (36) very accurately represents (37) for the real part of the field with lesser accuracy for the imaginary part of the field in a small neighborhood of $k_{x} / k_{0}>1$. Meanwhile, as $k_{0} L$ increases, a larger linear system size is needed, than that required for smaller $k_{0} L$, to represent (37) accurately. 


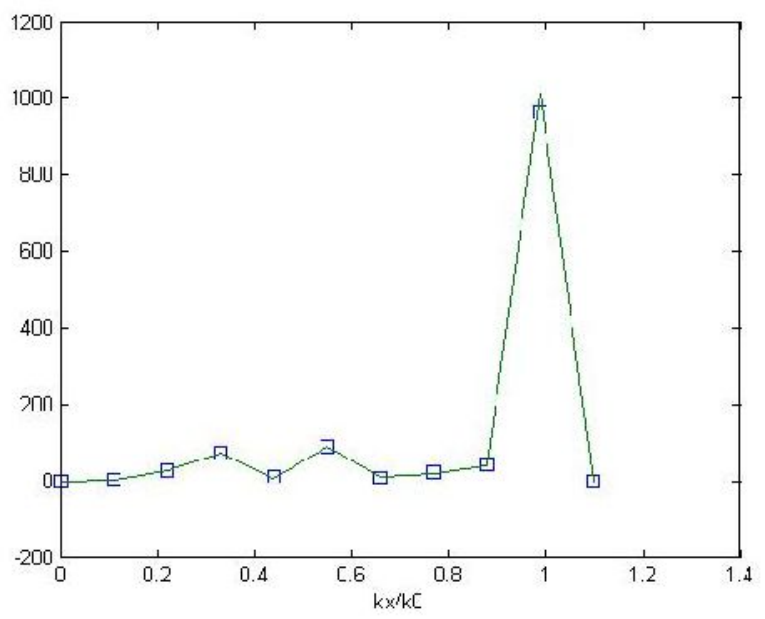

Figure (1): Real $E_{z r}\left(k_{x}, 0\right)$ for $k_{0} L=40$ and $N=1000$.

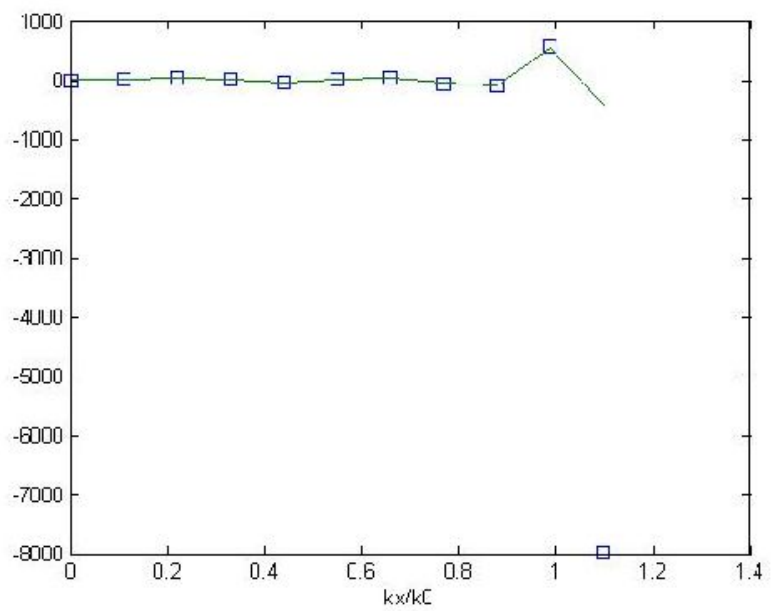

Figure (2): Imaginary $E_{z r}\left(k_{x}, 0\right)$ for $k_{0} L=40$ and $N=1000$. 


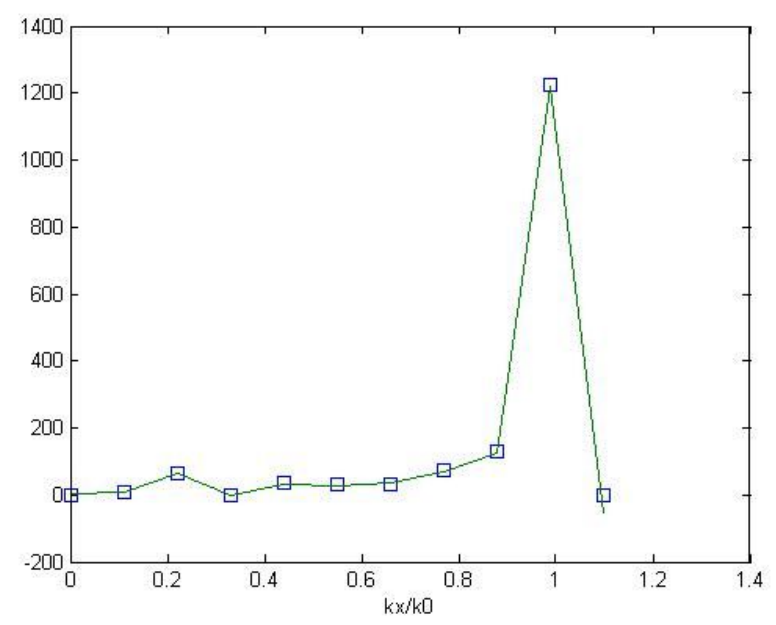

Figure (3): Real $E_{z r}\left(k_{x}, 0\right)$ for $k_{0} L=80$ and $N=1500$.

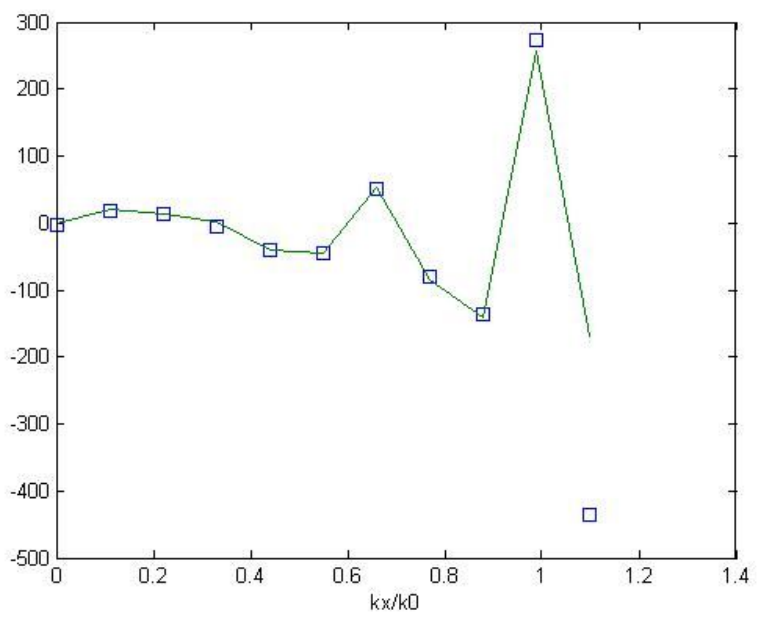

Figure (4): Imaginary $E_{z r}\left(k_{x}, 0\right)$ for $k_{0} L=80$ and $N=1500$. 


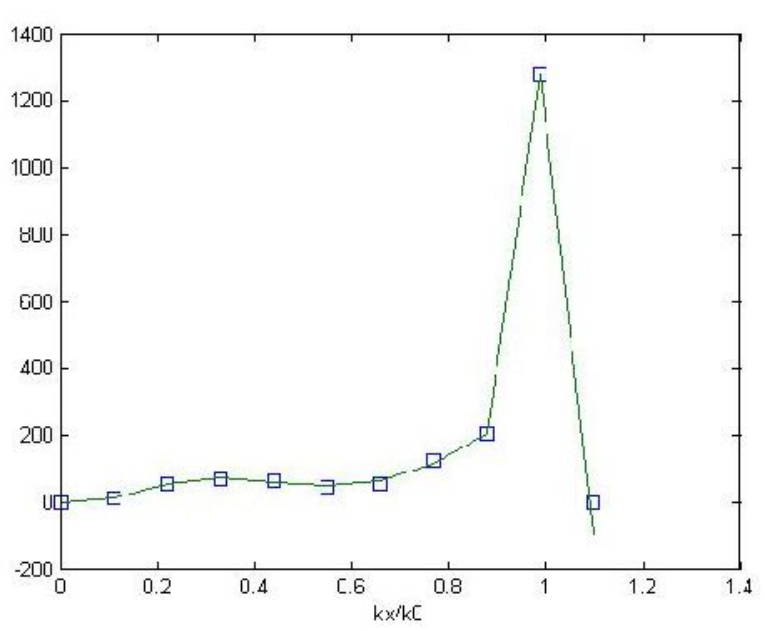

Figure (5): Real $E_{z r}\left(k_{x}, 0\right)$ for $k_{0} L=120$ and $N=1500$.

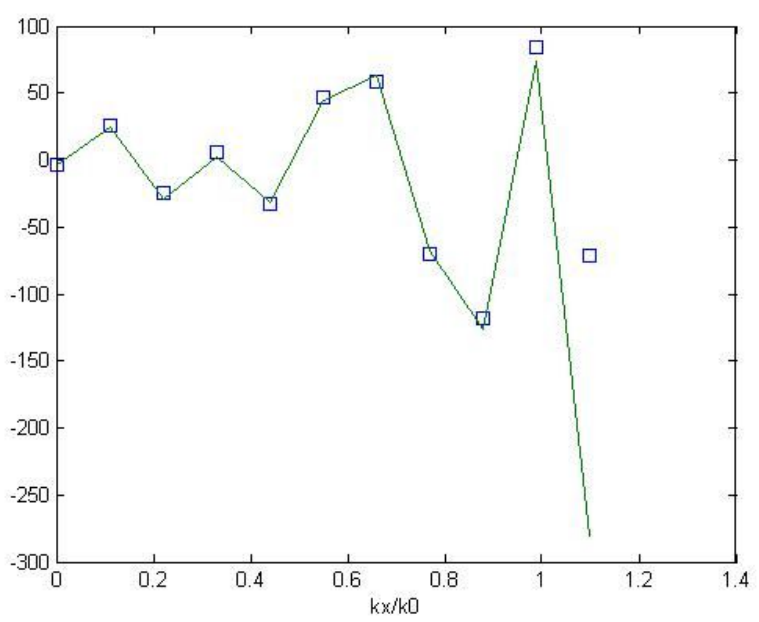

Figure (6): Imaginary $E_{z r}\left(k_{x}, 0\right)$ for $k_{0} L=120$ and $N=1500$.

\section{Conclusions:}

The proposed scheme proved to be very accurate and efficient for a wide range of problem parameters. It belongs to the class of schemes that can be divided into two parts. One that is independent of the properties of the scattering medium, hence, the current complete orthogonal set as well as the corresponding fields are computed once for all ranges of values of the medium parameters (this part is called Compute Once Use Many (COUM)). The second part is a very fast computation that produces the fields for a particular choice of medium parameters (this part is called Use Many (UM)). This characteristic of the scheme COUM/UM puts it far ahead, in terms of efficiency, of the 
schemes available in the literature where all of those are characterized by a Compute Many Use Many (CMUM), that is, a change in any problem parameter requires recomputing from the first step. This COUM/UM characteristic is especially crucial for problems wherein one needs to calculate many instances of the reflected fields for different values of medium parameters, for instance inverse scattering problems [11]. While we have presented the case of vertical stratification; extensions to include lateral variations are in progress.

\section{References:}

[1] A. Taflove and S. C. Hagness, Computational Electrodynamics: The FiniteDifference Time-Domain Method, $3^{\text {rd }}$ ed., Artech House, 2005.

[2] J. Jin, The Finite Element Method in Electromagnetics, $2^{\text {nd }}$ ed., John Wiley \& Sons, 2002.

[3] F. París and J. Cañas, Boundary Element Method: Fundamentals and Applications, Clarendon Press, 1997.

[4] J. L. Volakis and K. Sertel, Integral Equation Methods for Electromagnetics, SciTech Publishing, Inc., 2012.

[5] L. Felsen and N. Marcuvitz, Radiation and Scattering of Waves, IEEE Press, 1994, pp. 274-278.

[6] A. J. Devaney and E. Wolf, "Radiating and nonradiating classical current distributions and the fields they generate," Phys. Rev. D Part. Fields, 8, pp. 10441047, 1973.

[7] T. M. Habashy, M. L. Oristaglio and A. T. de Hoop, "Simultaneous nonlinear reconstruction of two-dimensional permittivity and conductivity," Radio Science, 29 (4), pp. 1011-1118, 1994.

[8] A. Gamliel, K. Kim, I. Nachman and E. Wolf, "A new method for specifying nonradiating monochromatic scalar sources and their fields," Journal of the Optical Society of America A, 6, pp. 1388-1393, 1989.

[9] E. Wallacher and A. K. Louis, "Complete sets of radiating and nonradiating parts of a source and their fields with applications in inverse scattering limited-angle problems," International Journal of Biomedical Imaging, 2006, p. 93074, 2006.

[10] E. A. M. ElFayome, Solutions of Microwave Problems Using VIE, M.Sc. Thesis, in progress, Electronics and Communications Department, Faculty of Engineering, Ain Shams University, Egypt.

[11] E. A. M. ElFayome, A. H. Kamel, and H. S. ElHennawy, "Application of radial neural networks for depth profile inversion," ICMA15, December 27-29, 2015, Egypt. 